\title{
Probing near-solid density plasmas using soft X-ray scattering
}

\author{
S Toleikis ${ }^{1}$, T Bornath ${ }^{2}$, T Döppner ${ }^{3}$, S Düsterer ${ }^{1}, \mathbf{R}$ R \\ Fäustlin ${ }^{1}$, E Förster ${ }^{4}$, C Fortmann ${ }^{5}$, S H Glenzer ${ }^{3}$, S \\ Göde $^{2}$, G Gregori ${ }^{6}$, R Irsig ${ }^{2}$, T Laarmann ${ }^{1}$, H J Lee ${ }^{7}$, B \\ $\mathbf{L i}^{8}, \mathbf{K}-\mathbf{H}$ Meiwes-Broer ${ }^{2}$, J Mithen ${ }^{6}, \mathbf{B}$ Nagler $^{7}$, A \\ Przystawik $^{2}$, P Radcliffe ${ }^{9}, \mathbf{H}$ Redlin $^{1}, \mathbf{R}$ Redmer $^{2}, \mathbf{H}$ \\ Reinholz $^{2}$, G Röpke ${ }^{2}, \mathbf{F}$ Tavella ${ }^{1}$, R Thiele $^{2}$, J \\ Tiggesbäumker ${ }^{2}$, I Uschmann ${ }^{4}$, S M Vinko ${ }^{6}$, T Whitcher ${ }^{6}$, \\ U Zastrau $^{4}$, B Ziaja ${ }^{10,11}$ and T Tschentscher ${ }^{9}$ \\ ${ }^{1}$ Deutsches Elektronen-Synchrotron DESY, Notkestr. 85, 22607 Hamburg, \\ Germany \\ ${ }^{2}$ Institut für Physik, Universität Rostock, Universitätsplatz 3, 18051 Rostock, \\ Germany \\ ${ }^{3}$ Lawrence Livermore National Laboratory, 7000 East Av., Livermore, CA \\ 94550, USA \\ ${ }^{4}$ Institut für Optik und Quantenelektronik, Friedrich-Schiller-Universität Jena, \\ Max-Wien-Platz 1, 07743 Jena, Germany \\ ${ }^{5}$ Department of Physics and Astronomy, University of California, Los Angeles, \\ CA 90095, USA \\ ${ }^{6}$ Department of Physics, Clarendon Laboratory, University of Oxford, Parks \\ Road, Oxford, OX1 3PU, UK \\ 7 SLAC National Accelerator Laboratory, 2575 Sand Hill Road, Menlo Park, CA \\ 94025, USA \\ ${ }^{8}$ Central Laser Facility, Rutherford Appleton Laboratory, Didcot, OX11 0QX, \\ UK \\ ${ }^{9}$ European XFEL GmbH, Albert-Einstein-Ring 19, 22671 Hamburg, Germany \\ 10 CFEL, Notkestr. 85, 22607 Hamburg, Germany \\ 11 Institute of Nuclear Physics, Radzikowskiego 152, 31-342 Krakow, Poland \\ E-mail: sven.toleikis@desy.de
}

\begin{abstract}
.
X-ray scattering using highly brilliant X-ray free-electron laser (FEL) radiation provides a new access to probe free electron density, temperature and ionization in near-solid density plasmas. First experiments at the soft X-ray FEL FLASH at DESY, Hamburg, show the capabilities of this technique. The ultrashort FEL pulses in particular can probe equilibration phenomena occurring after excitation of the plasma using ultrashort optical laser pumping. We have investigated liquid hydrogen and find that the interaction of very intense soft $\mathrm{X}$-ray FEL radiation alone heats the sample volume. As the plasma establishes, photons from the same pulse undergo scattering, thus probing the transient, warm dense matter state. We find a free electron density of $(2.6 \pm 0.2) \cdot 10^{20} \mathrm{~cm}^{-3}$ and an electron temperature of $14 \pm 3.5 \mathrm{eV}$. In pump-probe experiments, using intense optical laser pulses to generate more extreme states of matter, this interaction of the probe pulse has to be considered in the interpretation of scattering data. In this paper we present details of the experimental setup at FLASH and the diagnostic methods used to quantitatively analyze the data.
\end{abstract}


PACS numbers: 41.60.Cr

Free-electron lasers

PACS numbers: 52.20.Fs

Electron collisions

PACS numbers: 52.25. Os

Emission, absorption, and scattering of electromagnetic radiation

PACS numbers: 52.50.Jm

Plasma production and heating by laser beams 


\section{Introduction}

Emerging free-electron lasers (FEL) in the XUV to soft X-ray [1] and in the hard $\mathrm{X}$-ray [2] regimes provide radiation properties enabling scientific applications which are not feasible using synchrotron radiation. One of these new applications is the investigation of short-living, highly excited states of matter, here of near-solid density plasma states. These plasmas are often produced by exciting solid matter using ultrashort pulse optical laser radiation. Typical excitation time constants are in the fs to ps time domain while the expansion of the plasma state occurs on the ns time scale. The sub-picosecond pulse duration and high pulse intensity of FEL radiation provide excellent conditions to study the formation, evolution and relaxation of such plasma using either single-shot or accumulated measurements.

The experimental investigation of dense plasmas with temperatures of the order of $\mathrm{eV}(\approx 12000 \mathrm{~K})$ up to $\mathrm{keV}$ and pressures up to the Gbar regime using X-ray radiation is an area of active research [3] and provides a number of experimental challenges. $\mathrm{X}$-ray frequencies are required to probe dense plasmas since the frequency of the probing radiation $\omega_{\text {rad }}$ needs to be significantly larger than the density dependent plasma frequency $\omega_{\mathrm{p}}=\sqrt{\mathrm{e}^{2} n_{\mathrm{e}} / \epsilon_{0} m_{\mathrm{e}}}$. The results of such experiments deepen the understanding of the physical processes occurring in such highly excited matter and serve also to benchmark theoretical modeling and simulations. An area of particular interest is that of warm dense matter (WDM). This regime between the degenerate, strongly coupled condensed matter and the ideal plasma states presents a difficulty to theoretical and experimental access [4]. A systematic understanding of this largely unknown WDM domain is crucial, e.g. for the modeling of plasma generation, for example in laser shock-wave or inertial confinement fusion (ICF) experiments, or for astrophysical models, like for example the interior of giant planets. Experimental access to the WDM regime is restricted due to the need to achieve well-defined plasma conditions in terms of electron density and temperature.

In this work we report on experiments using the technique of spectrally resolved X-ray scattering, so-called X-ray Thomson scattering [5]. Thomson scattering using optical radiation is an extremely powerful technique to study dilute plasmas [6]. Transferring this technique to the X-ray regime provides a new diagnostic method allowing the determination of important plasma parameters such as temperature, density, and composition in dense plasmas $[5,7,8]$. Initial experiments showed the possibility to use non-collective [9] as well as collective X-ray Thomson scattering [10] to study WDM plasma properties. These initial X-ray experiments used backlighter sources, like proposed for ICF related research [11]. The development of FEL facilities for XUV up to hard X-ray radiation provides much more brilliant sources enabling new experimental access for WDM research. One example is to utilize the ultrashort FEL radiation for investigations of fs to ps time-dependent phenomena in plasmas using pump-probe techniques. While in the astrophysical context WDM states exist in a stable phase, in laboratory experiments they occur as transient states following nonequilibrium paths in the phase diagram. Hence, the extraction of accurate equation-ofstate (EOS) data requires the investigation of relaxation and equilibration dynamics of matter which is heated with intense femtosecond optical laser or soft X-ray FEL pulses. In general, the cold sample system is excited into a non-equilibrium, twotemperature state with $T_{\text {ion }} \ll T_{\text {electron }}$ immediately after heating $(<50 \mathrm{fs}$ ). In a next step the system develops into a local thermal equilibrium WDM state (after 1-2 ps) from where it further cools down by isentropic expansion ( $>2 \mathrm{ps}$ after irradiation). 


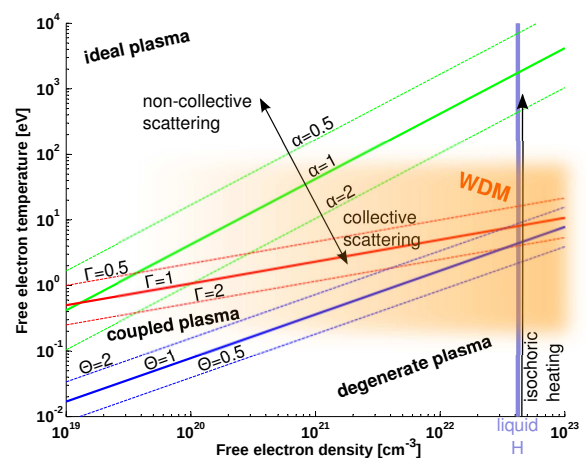

Figure 1. Phase space representation for electron density $n_{\mathrm{e}}$ and temperature $T_{\mathrm{e}}$. Lines for the scattering parameter $\alpha$, the coupling parameter $\Gamma$ and the degeneracy parameter $\Theta$ have been calculated according to [13]. The coupling parameter $\Gamma$ is defined as the ratio of the Coulomb energy between two charged particles and the thermal energy. It divides the phase space between ideal $(\Gamma \ll 1)$ or weakly coupled $(\Gamma \leq 1)$ plasmas and strongly coupled $(\Gamma \gg 1)$ plasmas. The degeneracy parameter $\Theta$, which is the ratio of thermal energy and the Fermi energy, divides the phase space into areas where quantum effects are of importance $(\Theta<1$, degenerated plasmas) and areas where the role of quantum effects decreases $(\Theta>1)$. The density of liquid hydrogen and the WDM regime are indicated. Via isochoric heating and the isentropic expansion mainly the phase space in the collective scattering regime is accessible.

Due to its ultra-short pulse duration $(\sim 15-50 \mathrm{fs})$ and high penetration power, X-ray FEL radiation is well suited for experiments probing these states in a time-resolving manner.

We report in this paper on experiments carried out at the first operating short-wavelength FEL facility FLASH (Free-electron LASer in Hamburg) at DESY, Hamburg. The self-amplified spontaneous emission principle [12] for generating FEL radiation in the XUV to soft X-ray wavelength range from 6.5 to $60 \mathrm{~nm}$ was first shown at FLASH [1]. Pulse durations of 15 to $50 \mathrm{fs}$ and pulse energies up to 150 $\mu \mathrm{J}$ are delivered for user experiments. Multiple pulses can be combined with variable spacing ranging from 1 to $25 \mu \mathrm{s}$ to form pulse trains which repeat every $0.2 \mathrm{~s}$. With these parameters FLASH reaches a peak brilliance of $10^{29}-10^{30}$ photons/ $\left(\mathrm{s} \mathrm{mrad}{ }^{2}\right.$ $\mathrm{mm}^{2} 0.1 \% \mathrm{BW}$ ). Using $13.5 \mathrm{~nm}$ FEL radiation, corresponding to a photon energy of $92 \mathrm{eV}$, we obtain access to near-solid density systems $\left(n_{\mathrm{e}}=10^{20}-10^{22} \mathrm{~cm}^{-3}\right)$ [13]. Future experiments using hard X-ray FELs, e.g. LCLS [2] or the European XFEL [14], will enable the investigation of plasmas at densities up to $n_{\mathrm{e}}=10^{26} \mathrm{~cm}^{-3}$, e.g. shockcompressed plasmas. Utilizing the high pulse energies we find that in experiments without optical laser excitation a significant portion of the FEL pulse interacts with the sample volume and generates a plasma while the other portion scatters from the heated system. Using this self-scattering scheme we were able to determine thermodynamic properties of the XUV heated hydrogen plasma at ultra-short time scales [15].

For the Thomson scattering experiments described here, cryogenic liquid hydrogen with an atom density of $n=4.2 \cdot 10^{22} \mathrm{~cm}^{-3}$ has been chosen as a sample. Hydrogen is currently in the focus of a world-wide effort to determine its EOS using various methods, facilities and theoretical approaches. Accurate EOS data are needed, amongst others, to develop realistic models for the interior of giant planets such as 


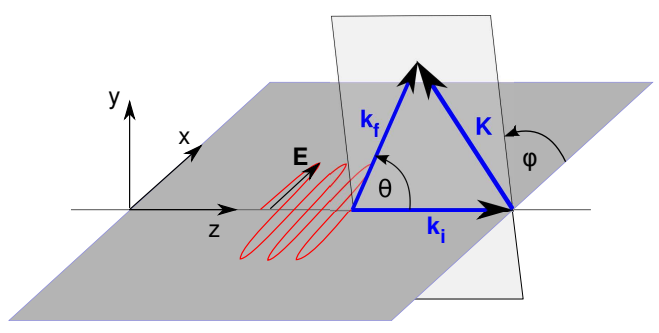

Figure 2. Scattering geometry at FLASH. The FEL beam propagates in the $\mathrm{z}$-direction and is horizontally polarized (x-direction).

Jupiter. Standard three-layer structure models assume a molecular non-conducting outer fluid layer, a conducting fluid layer below, and a possibly solid core of rocks and/or ices in the centre [16]. The chemical composition throughout the layers, the location of the layer boundaries, and the size of the core are adjusted to reproduce the observational constraints (given mass, radius, gravitational moments, mean helium fraction etc.). However, a complete verification of the planetary structure model is still missing and requires experimental data for the $\mathrm{H}$ and He EOS. A first interior structure model based almost entirely on finite-temperature density functional theory molecular dynamics (FT-DFT-MD) EOS data for $\mathrm{H}$ and He [17] yields significant modifications compared with results derived from chemical EOS models [18]. Experimental data for thermodynamic properties and structure factors data, see e.g. [8], could be tested against different EOS calculations (chemical picture [19] or FT-DFT-MD [20]). Due to the isochoric heating mechanism, adopted in our experiments, and the subsequent isentropic expansion, EOS data can be extracted especially for the WDM region of hydrogen at liquid density and below (see Fig. 1).

The following Section 2 will shortly describe the underlying experimental technique for Thomson scattering. In Sec. 3 the experimental setup and important methods will be described in detail. Also the main diagnostic tools are introduced, especially the soft X-ray spectrometers that have been used for Thomson scattering. In Sec. 4 current achievements will be discussed. Conclusions and future extension of this method will be summarized in Sec. 5 .

\section{Experimental technique}

It is conceptually straightforward to implement the Thomson scattering technique using the new FEL sources with the advantage of higher repetition rates, a high temporal resolution in the femtosecond regime, and a wide parameter range that is accessible with freely tunable FEL sources. The kinematics of the scattering process is determined by the momenta of the incoming X-ray photon, $\mathbf{k}_{\mathbf{i}}$, and the scattered photon, $\mathbf{k}_{\mathrm{f}}$, where the momentum $\mathbf{K}=\mathbf{k}_{\mathrm{i}}-\mathbf{k}_{\mathrm{f}},|\mathbf{K}|=k=\sqrt{k_{\mathrm{i}}^{2}+k_{\mathrm{f}}^{2}-2 k_{\mathrm{i}} k_{\mathrm{f}} \cos \theta}$ is transferred to the scattering particle [13]. The scattering geometry is shown in Fig. 2. The scattered power $P_{\mathrm{S}}$ from the electrons into a frequency interval $\mathrm{d} \omega$ and solid angle $\mathrm{d} \Omega$ is given by [23]

$$
P_{\mathrm{S}}(\mathbf{R}, \omega) \mathrm{d} \Omega \mathrm{d} \omega=\frac{P_{\mathrm{i}} \mathrm{r}_{0}^{2} \mathrm{~d} \Omega}{2 \pi A}\left|\widehat{\mathbf{k}_{\mathrm{f}}} \times\left(\widehat{\mathbf{k}_{\mathrm{f}}} \times \widehat{\mathbf{E}}\right)\right|^{2} N S(\mathbf{k}, \omega) \mathrm{d} \omega
$$


Here $P_{\mathrm{i}}$ denotes the incident FEL power, $\mathrm{r}_{0}$ is the classical electron radius, $A$ the plasma area irradiated, $N$ the number of nuclei, and $S(\mathbf{k}, \omega)$ the total electron dynamical structure factor. The polarization term $\left|\widehat{\mathbf{k}_{\mathrm{f}}} \times\left(\widehat{\mathbf{k}_{\mathrm{f}}} \times \widehat{\mathbf{E}}\right)\right|^{2}$ reflects the dependence of the scattered power on the incident laser polarization with the hat denoting unit vectors. In case of linear polarization and for unpolarized light, this term is given by

$$
\left|\widehat{\mathbf{k}_{\mathrm{f}}} \times\left(\widehat{\mathbf{k}_{\mathrm{f}}} \times \widehat{\mathbf{E}}\right)\right|^{2}= \begin{cases}\left(1-\sin ^{2} \theta \cos ^{2} \varphi\right) & \text { lin. polarized } \\ \left(1-\frac{1}{2} \sin ^{2} \theta\right) & \text { unpolarized }\end{cases}
$$

As seen from Eq. 2, in case of linear polarization, the preferred scattering geometry is at an azimuth angle of $\varphi=90^{\circ}$, where the polarization term is getting independent of the scattering angle $\theta$.

The scattering kinematics determines if collective electron excitations or noncollective single electron properties are probed. To distinguish the two regimes the scattering parameter $\alpha=1 /\left(k \lambda_{\mathrm{D}}\right)$ is used. $\alpha>1$ corresponds to the collective regime where the probed length scales are larger than the Debye screening length $\lambda_{\mathrm{D}}^{2}=\epsilon_{0} \mathrm{k}_{\mathrm{B}} T /\left(n_{\mathrm{e}} \mathrm{e}^{2}\right)$. Here, $\mathrm{k}_{\mathrm{B}}$ is the Boltzmann constant and $\mathrm{e}$ is the charge of the electron. In this case scattering from collective electron excitations, i.e. plasmons, can be observed. In the non-collective regime, $\alpha<1$, the probed length scale is shorter than $\lambda_{\mathrm{D}}$ and the momentum distribution for individual electrons is probed. In Fig. 1 the separation lines $(\alpha=0.5 ; 1 ; 2)$ are drawn for $92 \mathrm{eV}$ photon energy and $\theta=90^{\circ}$. One observes that for these conditions primarily collective scattering is accessible. This collective scattering probes the existence of plasmons which are down- and up-shifted in energy, relative to the elastic scattering at the incoming photon energy. From the energy difference of these resonances with respect to the FEL probe pulse the electron density can be estimated. The slope of the peak heights of the resonances allows an independent estimate of the electron temperature [13]. In the non-collective scattering case, the inelastic portion of the scattered spectrum is dominated by Compton scattering. But since the energy transfer in the soft $\mathrm{X}$-ray regime is very small, typically below the energy resolution, it is difficult to investigate this non-collective regime with the available wavelength range at FLASH. Experimentally the elastic and inelastic scattered X-ray are measured as function of the time delay between heating (pump) and probe pulse. The measured spectrum is described by the dynamic structure factor $S(k, \omega)[24]$ in Eq. 1:

$$
S(k, \omega)=Z_{\mathrm{f}} S_{\mathrm{ee}}(k, \omega)+Z^{2}\left(1-e^{-2 W}\right) S_{\mathrm{ii}}(k, \omega) .
$$

Here, $Z$ is the nuclear charge and $Z_{\mathrm{f}}$ the average number of free electrons per atom. The first term describes the high-frequency free electron fluctuations which include inelastic collective plasmon scattering. The second term describes the ionic, nearly elastic response with the ion-ion structure factor $S_{\mathrm{ii}}$ modulated by Debye-Waller effects.

Using calculations for the dynamical structure factor $S(k, \omega)$ for a dense hydrogen plasma at thermal equilibrium $\left(T_{\mathrm{e}}=T_{\mathrm{i}}=12 \mathrm{eV}\right)$ we calculated Thomson scattering spectra (Fig. 3) as a function of the energy shift for three different FEL probe wavelengths $(25,13.5$ and $6 \mathrm{~nm})$ and for three different electron densities $(1 \times, 5 \times$ and $\left.10 \times 10^{21} \mathrm{~cm}^{-3}\right)$. Experimental resolution, mainly due to FEL bandwidth and final spectrometer resolution, is included in the simulated spectra by convolution with 


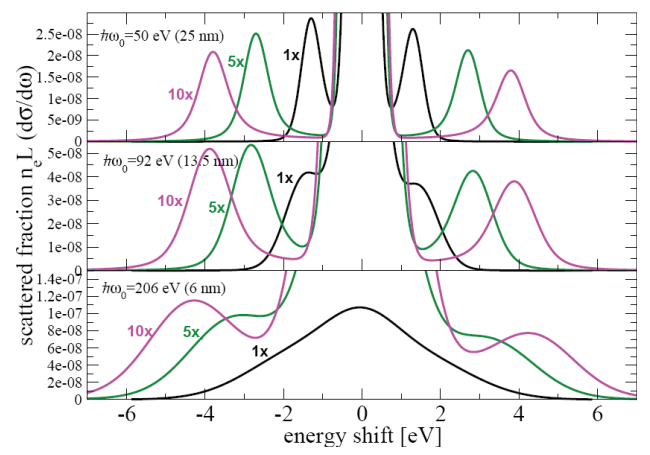

Figure 3. Calculated Thomson scattering spectra for three different FEL probe wavelengths $(25,13.5$ and $6 \mathrm{~nm})$. Each panel shows the scattered fraction of incoming photons for different electron densities $n_{\mathrm{e}}$ in units of $10^{21} \mathrm{~cm}^{-3}$, at equilibrium conditions $T_{\mathrm{e}}=T_{\mathrm{i}}=12 \mathrm{eV}$, an ionization degree $Z_{\mathrm{f}}=1$ and a scattering angle of $\theta=90^{\circ}$. The spectra are convoluted with an expected finite bandwidth of $\sim 1 \%$ of the incoming FEL radiation.

a Gaussian profile of $\delta \omega / \omega=0.01$ full width half maximum (FWHM). In Fig. 3 we find the ion feature, i.e. quasi elastic scattering by bound electrons, at zero energy shift. Furthermore, up- and down-shifted plasmon resonances are observed at energies near the plasma frequency $\omega_{\mathrm{p}}$ which is related to the free electron density. Although the plasmons are much easier to resolve at a longer wavelength $(25 \mathrm{~nm})$, the absolute number of scattered photons is less if one compares the absolute numbers for the scattered fraction of photons at different probing wavelengths. Using these simulations we chose $13.5 \mathrm{~nm}$ as the probe wavelength providing a reasonable compromise between resolution and scattered intensity.

Variation of the scattering angle gives different $\mathbf{K}$ and allows testing theoretical models for the structure factor, and EOS data can be determined, e.g. through the excess internal energy in the one component plasma approximation [42]

$$
P=n_{\mathrm{e}} \mathrm{k}_{\mathrm{B}} T_{\mathrm{e}}+\frac{n_{\mathrm{e}}}{12 \pi^{2}} \int \frac{Z^{2} \mathrm{e}^{2}}{k^{2}}\left[S_{\mathrm{ii}}(k)-1\right] d k .
$$

The electron density can be determined from the energy shift of the plasmon peaks in the scattered spectra thus providing a direct and independent measurement [13]. Similarly, the temperature is experimentally obtained from the intensity ratio of the up- and down-shifted plasmons, via the detailed balance relation [13]. An important point in extracting the EOS via Eq. 4 is the separation in energy between the two terms in Eq. 3, thus allowing a well defined determination of the ion-ion structure factor. Also, while the experiments are conducted at only a few representative $\mathbf{K}$ vectors, the integration of Eq. 4 requires knowledge of $S_{\mathrm{ii}}(k)$ over the whole range of scattering angles (or wavenumbers). This is achieved by matching the experimentally measured structure factor with theoretical curves obtained within a two component plasma hypernetted chain calculation (TCP-HNC) scheme, which is computationally very efficient by using effective electron-ion potentials extracted from FT-DFT-MD simulations [7]. 


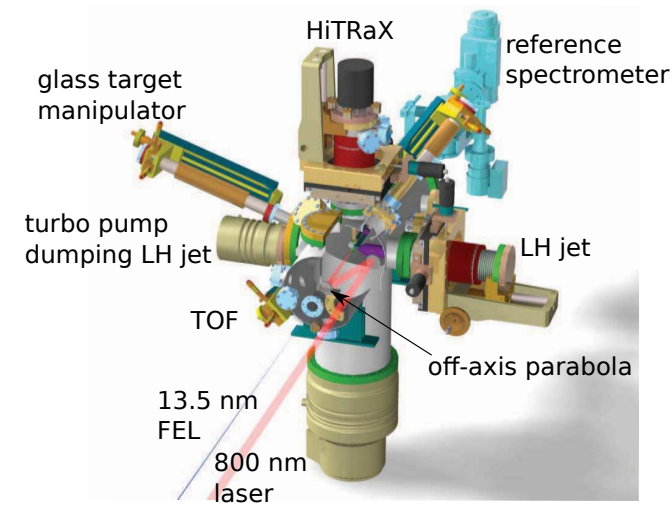

Figure 4. Experimental setup to study dense plasmas using Thomson scattering at FLASH.

\section{Experimental setup}

FLASH was operating at $92 \mathrm{eV}$ photon energy, corresponding to a photon wavelength of $13.5 \mathrm{~nm}$. The employed operation mode of FLASH has generated soft X-ray pulses with pulse durations of $15-50 \mathrm{fs}$, a bandwidth of $\sim 1.0 \%$, and pulse energies up to 150 $\mu \mathrm{J}$. The experimental vacuum chamber has been set up at beamline BL2, where the FEL beam was then focused to a $\sim 20-30 \mu \mathrm{m}$ (FWHM) focal spot by an ellipsoidal mirror at the end of the beamline. The overall beamline transmission is $64 \pm 4 \%$ [25].

The vacuum chamber with its main components is shown in Fig. 4. The FEL beam passes horizontally through the chamber aligned such that the focal point is located in the center of the chamber. At the focus the hydrogen pellet source horizontally injects a continuous liquid hydrogen jet or liquid hydrogen droplets depending on the operation mode. The details of this source are described in Sec. 3.2. The FEL beam at FLASH is horizontally polarized and in order not to decrease the scattered power the soft X-ray spectrometer is mounted vertically at a scattering angle of $\theta=90^{\circ}$. In addition a reference spectrograph is mounted in transmission direction to monitor the reference wavelength of the incident FEL beam for a later comparison with the scattering spectra recorded at $90^{\circ}$. Further diagnostics that have been employed in the vacuum chamber are an electron and ion time-of-flight (TOF) spectrometer, several CCD cameras and a long range microscope for monitoring the interaction region. The liquid hydrogen jet which is horizontally injected is dumped in a turbomolecular pump to reduce the gas ballast in the chamber. As the experimental chamber is directly connected to the beamline vacuum, additional differential pumping stages have been employed to allow secure operation of the hydrogen pellet source which under normal operation leads to pressures in the main chamber of up to several $10^{-4}$ mbar. The additionally employed differential pumping stage allows together with the beamline differential pumping stage to tolerate a pressure difference of more than two orders of magnitude. This is sufficient in order to safely connect the experiment to the beamline vacuum. The optical pump laser at $800 \mathrm{~nm}$ wavelength is guided and reflected onto an off-axis parabolic mirror which has a hole to let pass the FEL beam, allowing on the one hand to collinearly overlap both beams and on the other hand to focus the optical laser to same focal spot as the FEL beam. Details concerning the optical laser 
and the procedures to find spatial and temporal overlap with FEL beam are given in the following Sec. 3.1.

\subsection{Optical laser: spatial and temporal overlap}

For pump-probe experiments it is essential to have a very good spatial and temporal overlap of both the optical laser beam and the FEL beam. The location of the interaction is given by the focal length of the ellipsoidal beamline mirror of $2 \mathrm{~m}$ at beamline BL2. The optical laser is focused by an off-axis parabolic mirror collinearly to the same location with a slightly larger focal spot size. Spatial alignment is achieved by overlapping optical laser focal spot and FEL spot at reduced intensities on a cerium doped $\mathrm{Y}_{3} \mathrm{Al}_{5} \mathrm{O}_{12}$ (Ce:YAG) coated screen and observing the respective scattered or fluorescence signal with a long-range microscope. Temporal overlap with femtosecond time resolution is more difficult to obtain. In a first set of experiments we have successfully used the generation of side bands in electron time-of-flight spectra to determine and adjust the overlap of soft X-ray and optical pulses [26]. This method unfortunately constrains the setup of the experiment, as the turbomolecular pump where the hydrogen jet is dumped has to be replaced with a relatively large TOF setup to achieve sufficient high resolution in order to observe the side bands. To overcome these constraints, we successfully implemented a shadowgraphy technique. Therefore we position a movable glass slide in the target region, attenuate the optical laser with a neutral density filter of known thickness, and defocus by moving a lens already in the beam path. Using the FEL we excite the electron system of the glass leading to ultrafast modification of transmission at $800 \mathrm{~nm}$. If the plasma created by the FEL pulse is present when the optical laser pulse reaches the target, the optical laser pulse is reflected on the plasma critical surface. This effect can be observed in transmission. If the optical laser pulse arrives before the FEL pulse its transmission is not altered. Scanning the delay of the optical laser with respect to the FEL pulse while observing the transmitted signal allows us to find the temporal overlap. This process is illustrated in Figure 5. Since the FEL pulses lead to permanent damage in the glass distorting the light transmission, the glass target is mounted on a manipulator and shifted between pulses (see Fig. 4). To facilitate the alignment procedure, FEL and optical laser were operated with a fast shutter. When temporal overlap is found, the focusing lens is returned to its original position and the neutral density filter is removed. The optical laser delay is compensated for the thickness of the removed filter. The accuracy of this method is in our case limited by the pulse duration of the optical laser which was $\sim 150 \mathrm{fs}$, but also by the temporal jitter between FEL and optical laser pulses. A more detailed investigation of this shadowgraphy based method has been performed in a different experiment at FLASH [27]. Once temporal overlap is found it needs to be maintained as the electron bunch arrival time can vary, mainly due to variations in the electron trajectory in the accelerator. This is achieved using an optical streak camera continuously comparing the relative timing of optical laser light and optical bending magnet radiation arising from the last dipole magnet of the FLASH accelerator. Changes in relative arrival time are then compensated with the delay line of the optical laser. Further details of the FLASH pump-probe laser system are described in Ref. [25]. 


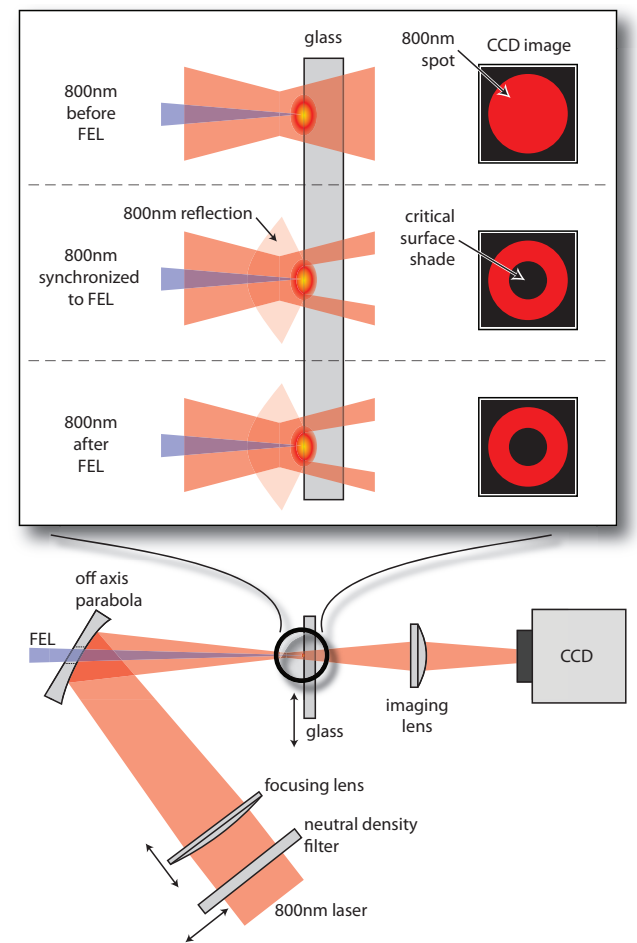

Figure 5. Measurement of the temporal overlap between FEL and optical laser pulses. The FEL pulse generates a plasma on a glass target and the plasma critical surface is imaged in transmission by backlighting with an optical laser pulse.

\subsection{Liquid hydrogen source}

To provide a near-solid density state of hydrogen in the focus without resorting to compounds the use of cryogenic liquid or solid hydrogen as a target is mandatory. The controlled production of microscopic streams of a material is an ongoing effort in several fields. Besides providing scattering targets for particle accelerators [28, 29], similar sources using xenon, water or liquid metal jets were investigated for generation of plasma EUV radiation [30].

Generally, the principle of operation is to press a fluid through a small orifice into the vacuum in a way that a continuous liquid filament is formed. Such a cylindrical liquid is unstable against its surface tension, small fluctuations will grow and the Rayleigh breakup process will produce almost monodisperse droplets with a size approximately 1.8 times the nozzle diameter. By introducing an artificial perturbance to the liquid, the breakup process can be stabilized and the tuning of the droplet size becomes possible. The common method is to vibrate the nozzle with a high frequency piezo actuator. Either way, the produced droplets freeze by evaporational cooling in the vacuum and form solid pellets after several millimeters in flight.

In our case, a temperature controlled helium flow cryostat is used to cool down a home-made nozzle holder and the hydrogen to the needed temperature in the range of $20 \mathrm{~K}$. A picture of the hydrogen liquid jet and pellet source and a sketch of the cold head are shown in Fig. 6. The cryostat is mounted on a three-dimensional manipulator 


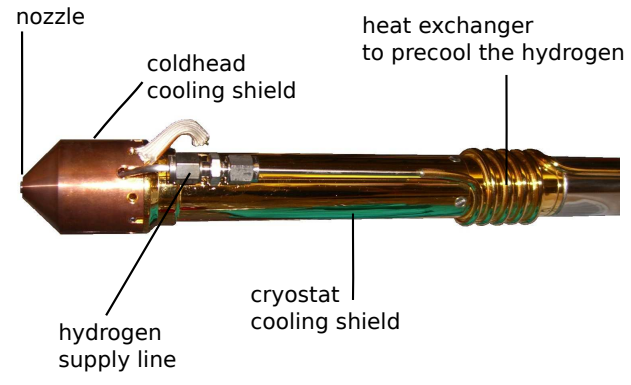

(a) Picture of the complete source

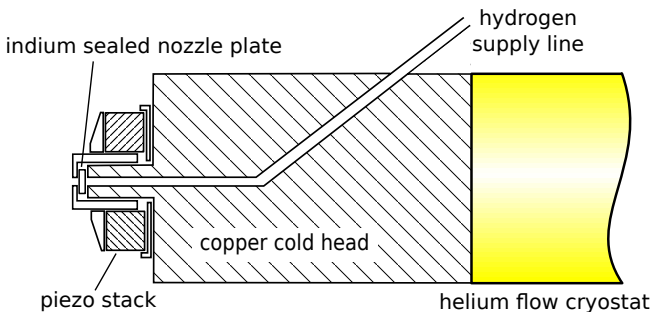

(b) Sketch of the cold head

Figure 6. The left figure (a) shows a picture of the hydrogen pellet source used to study WDM at FLASH. It utilizes a cryostat to control the temperature of a cold head holding the nozzle and the piezo transducers. Details of the cold head are shown in the right figure (b).

to provide the alignment to the focus area. Commercially available pinhole apertures for electron microscopes serve as nozzles and create a short channel, which is beneficial for suppressing turbulence in the nozzle. The necessary vacuum conditions in the chamber limit the maximum nozzle diameter to $20 \mu \mathrm{m}$. The hydrogen backing pressure in our experiment is typically 2 bar which results in a velocity of about $60 \mathrm{~m} / \mathrm{s}$ of the liquid filament. The source can be operated in different modes. At higher temperatures, smaller droplets in a divergent jet are generated. In the Rayleigh regime, a well collimated stream of droplets is produced. Here the piezo actuator can be used to modulate the filament with frequencies between 25 and about $100 \mathrm{kHz}$ which provides a limited size control and the possibility to synchronize the droplets in the focus area with the optical laser and the FLASH pulse. The timing synchronization with respect to generation of the droplets still needs to be determined. Furthermore, it proved hard to maintain the pointing stability of the cryogenic jet in order to place the hydrogen targets exactly in the focus of the optical laser, the FLASH beam and the soft X-ray spectrometer. Therefore it was necessary to monitor the spatial overlap with the FLASH beam on a shot by shot basis, see Sec. 3.4.

If the nozzle temperature is lowered further, the hydrogen solidifies before uniform droplets have formed. Eventually a continuous solid filament leaves the nozzle. This operating mode was not useful for our experiment with a horizontally mounted source. The results described in Sec. 4 are obtained while the source has been operated in the continuous liquid filament mode without using the piezo actuator. The chosen nozzle diameter of $15 \mu \mathrm{m}$ results in a filament diameter of $25-30 \mu \mathrm{m}$ and matches with the focal spot size of BL2. The absorption length for $92 \mathrm{eV}$ photons is $7 \mu \mathrm{m}$ [31]. Thus, with this sample size FLASH photons can deeply penetrate into the target. The interaction point has been set $\sim 1-2 \mathrm{~mm}$ away from the nozzle exit.

\subsection{Soft $X$-ray spectrometers}

During the first experiment a transmission grating spectrometer for soft X-rays has been used [32]. It was located at the same position as the later installed HiTraX spectrometer, see Fig. 4. The transmission grating spectrometer is composed of a nickel coated toroidal mirror, a 2000 lines $/ \mathrm{mm}$ free standing transmission grating, and a back-illuminated soft X-ray CCD. The toroidal mirror itself is a point to point 


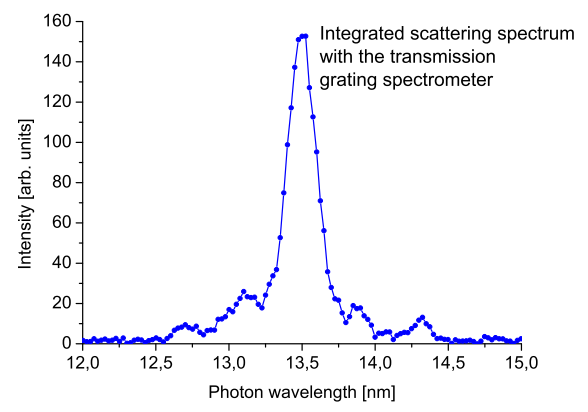

Figure 7. Sample scattering spectrum obtained using the transmission grating and clearly exhibiting artificial sidebands with a periodicity of $0.4 \mathrm{~nm}$. Integration time is 30 minutes (180000 pulses) at a mean FEL energy of $38.4 \mu \mathrm{J}$.

imaging system with a magnification $M=1$ and an image distance of $357 \mathrm{~mm}$. For such a system the incidence angle is about $86.14^{\circ}$, which is the total reflection angle of the nickel mirror. The transmission grating with a period of $a=200 \mathrm{~nm}$ consists of free-standing gold bars. The bars are intersected by a support grid with quadratic periods of $17 \mu \mathrm{m}$. The solid angle of acceptance is $\Delta \Omega \approx 4 \cdot 0 \cdot 10^{-4} \mathrm{sr}$ which is determined by a circular aperture with a diameter of $d=8 \mathrm{~mm}$, located in front of the mirror. The spectral range of the spectrometer is about 6-19 $\mathrm{nm}$. A sample scattering spectrum is shown in Fig. 7. In the spectrum the elastically scattered Rayleigh peak from the incident FEL pulse on cryogenic hydrogen is visible. In addition a sideband structure around the main elastic peak is seen. The reason for this sideband structure is that the support grid of the grating bars also acts as a 2-D transmission grating itself. First of all, the grid could result in a dispersion of visible light. This spectrum would then overlap with the soft X-ray spectrum. Using thin metal filters visible radiation is suppressed. Secondly, the grid can also diffract the soft X-ray radiation. This causes side peaks around the initial main peak. The 'wavelength shift' of the side peak is about $0.4 \mathrm{~nm}$. This is indeed confirmed by calculations. In addition, the width (FWHM) of the Rayleigh peak in the 1st order is broadened by $0.18 \mathrm{~nm}$ in comparison to the 0 th order, where the width (FWHM) is $0.12 \mathrm{~nm}$. This coincides with expected wavelength bandwidth of $\sim 1 \%$ of the FEL beam. This sideband structure inhibited the detection of plasmons in this first run.

Therefore a new compact and light weight soft X-ray spectrograph covering 5-35 $\mathrm{nm}$ and employing a toroidal mirror and a variable line space reflection grating has been developed [33]. This High $\underline{T h r o u g h p u t, ~ H i g h ~} \underline{R}$ esolution Spectrograph for Soft $\underline{X}-$ Ray Light (HiTRaX) has been employed in the latter Thomson scattering experiments. It provides a much larger solid angle of acceptance of $\Delta \Omega=1.9 \cdot 10^{-3}$ sr and a resolution of $\lambda / \Delta \lambda=330$ measured at $21 \mathrm{~nm}$. The improved overall detection efficiency can be seen in Fig. 8 where a scattering spectrum has been recorded with only one single FEL pulse hitting the cryogenic hydrogen target. The astonishing improvement in detection efficiency should be only a factor of $\sim 10$ at $13.5 \mathrm{~nm}$ if one calculates the theoretical throughput including all components in both spectrometers [33]. Here the conclusion is that the toroidal mirror of the transmission grating spectrometer that has been used in the first run had a much worse reflection efficiency than initially assumed due to accidentally coated impurities of the mirror. 


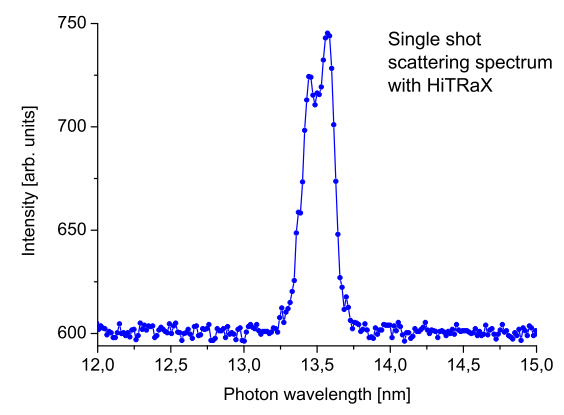

Figure 8. Single shot spectrum scattering from cryogenic hydrogen measured with the HiTRaX spectrometer at a FEL pulse energy of $78 \mu \mathrm{J}$.

In addition to the spectrometer that detects Thomson scattering we have also employed another soft X-ray spectrometer which serves as reference spectrometer to measure the incoming FEL radiation. This spectrograph is based on a cylindrically bent variable line space grating (average density 1200 lines $/ \mathrm{mm}$ ) in gracing incidence geometry [34]. It was located $\sim 170 \mathrm{~cm}$ behind the interaction region (see Fig. 4) and detects part of the FEL beam which passes by the liquid hydrogen target as the FEL focus is slightly larger than the hydrogen target. Because of the high intensity it was necessary to introduce $1 \mu \mathrm{m}$ mylar filter foil in front of the spectrometer to reduce the transmission. A slit further reduces the transmission, acts as a differential pumping stage and finally defines the achievable resolution of the spectrometer, which has been determined to $\lambda / \Delta \lambda \approx 200$ from single shot spectra. The idea of the reference spectrometer is to use the recorded reference spectra as a kernel to deconvolute the Thomson scattering signal. In the analysis it turned out that spectra with longer integration times revealed substantially lower resolution which we accounted to mechanical vibrations of the support structure of the spectrometer. Thus a deconvolution of the Thomson scattering spectra was not possible.

In future, because of the improved photon diagnostic capabilities of the FLASH facility, the use of a reference spectrograph will become obsolete, as this new diagnostic devices will provide spectral information of each FEL pulse on a shot-to-shot basis for the users.

\subsection{Time-of-flight spectrometer}

A field free time-of-flight spectrometer was fitted to the experiment. The initial idea was to determine the kinetic energy of electrons emanating from the dense plasma as well as recoil energy of ions. It turned out that the used micro channel plate detector (MCP) of the TOF was very sensitive to soft X-ray photons. The time resolution of the MCP allows a convenient discrimination between a prompt peak caused by elastically scattered X-ray photons and the later arriving electrons. This is shown in Fig. 9. The integral light signal is proportional to the amount of photons impinging the MCP. On top of that, an electron signal is visible $\sim 2 \cdot 10^{-7} \mathrm{~s}$ after the light peak. The MCP was located approximately $70 \mathrm{~cm}$ from the interaction region, so that the electrons have a kinetic energy of $\sim 35 \mathrm{eV}$. The electron signal can be identified by switching off the Helmholtz coils so that the charged particles are dumped in the 


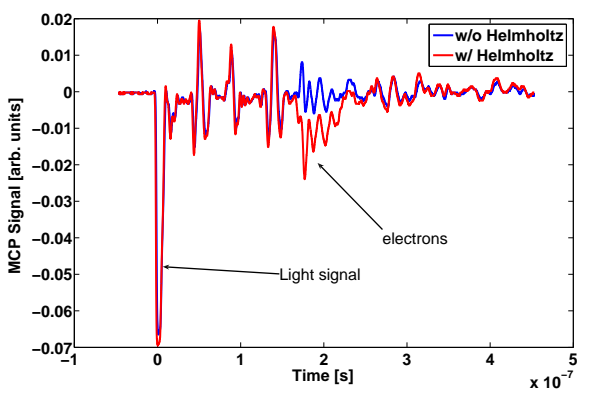

Figure 9. Typical TOF single shot spectrum from the interaction of an intense FEL pulse with the hydrogen sample showing a prompt X-ray scattering feature and signals from electrons emitted from the plasma. The latter can be identified through the Helmholtz coils that compensate for the Earths magnetic field. The additional oscillations are caused by electronic noise of the read out system.

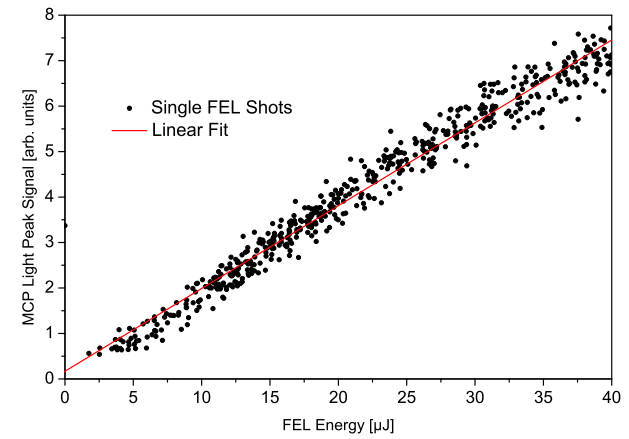

(a) Homogenous Xe gas

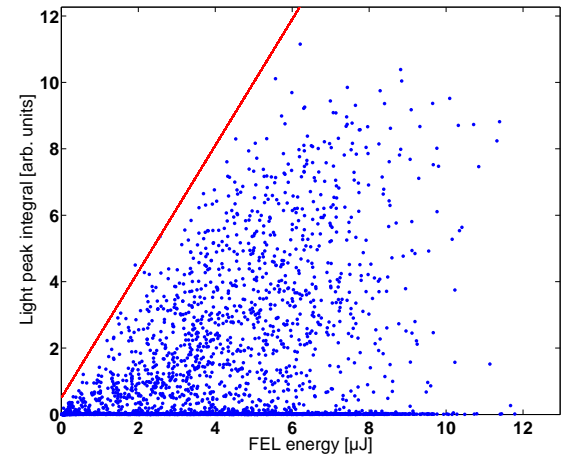

(b) Cryogenic $\mathrm{H}$ jet

Figure 10. Correlation of the prompt X-ray peak detected by the MCP of the TOF versus the FEL pulse energy detected by the gas monitor detector (GMD) of FLASH. The left plot shows the expected linear correlation for the scattered signal from atomic xenon gas. The right plot shows the distribution for X-ray scattering from the spatially unstable hydrogen target.

wall of the vacuum chamber due to their curved trajectory in the earths magnetic field. The integral of the prompt signal by X-rays correlates linearly with the incident FEL intensity measured with the gas monitor detector (GMD) system of FLASH [25]. This is shown in Fig. 10(a), where the correlation on a pulse to pulse basis between the integral of the light peak and the FEL pulse energy is plotted for scattering from an homogenous xenon gas target. In comparison to the measurement with xenon gas, the respective plot for scattering from liquid hydrogen looks very different. This is shown in Fig. 10(b). Here most data points do not correlate linearly with the FEL pulse energy, most probably due to the spatial instability of the liquid hydrogen source. The measured distribution reveals that a lot of FEL pulses did not hit the hydrogen target at all, i.e. no scattered light was detected by the MCP detector. In addition, many pulses yield a weak scattering signal, while only few FEL pulses have produced a maximum scattering signal. These maximum signals indeed correlate with 
the FEL pulse energy indicated by a red line in Fig. 10(b). The ratio of the actual scattered intensity versus the expected maximal scattered intensity (red line) gives a quantitative estimate of the spatial overlap between FEL beam and the hydrogen target on a single pulse basis.

\section{Results and discussion}

Experiments have been carried out both in optical laser pump soft X-ray probe and in soft X-ray only configurations. In the later configuration, also called self-Thomson scattering, the interaction of the soft X-ray FEL with the cold sample volume leads to plasma formation while simultaneously scattering occurs from the transiently changing system. The following discussion of current results concentrates on the analysis of spectra obtained in the self-Thomson scattering case and on their interpretation. Measurements of soft X-ray Thomson scattering spectra from optical laser heated liquid hydrogen plasma are still under evaluation.

For the self-Thomson scattering case a spectrum for 15 minutes integration time (4500 FEL pulses at an average intensity of $15 \mu \mathrm{J})$ is shown in Fig. 11. The spectrum shows equally red and blue shifted peaks from electronic plasma resonances, i.e. the plasmons. This observation in itself is very promising as the Thomson scattering integrates over all electrons in space and time inside the sample volume. The observation of plasmons indicates that a free electron plasma with electron equilibration was generated within the pulse duration of $40 \mathrm{fs}$. Furthermore, the scattering from these plasmon excitations is the major contribution to the overall scattering. It should be added here that the obtained scattering spectra reveal an unprecedented signal to noise ratio due to the high repetition rate of the FEL compared to single laser pulse driven X-ray sources $[8,9,10]$.

In preparative simulations we showed that plasmons can be resolved despite slight density and temperature gradients that are produced by FEL heating [35]. More in depth simulations take into account the temporal duration of the FLASH pulse as well as ultra short time scale interactions, in particular impact ionization $[15,39]$.

The quantitative analysis of the spectra uses the photon energy shift of the plasmons with respect to the incident radiation for the determination of the plasma free electron density via the Bohm-Gross dispersion relation [36]. We obtain a density of $n_{\mathrm{e}}=(2.6 \pm 0.2) \cdot 10^{20} \mathrm{~cm}^{-3}$, which corresponds to $\sim 1 \%$ ionization in the sample. The free electron temperature is determined through the intensity ratio of the two plasmon peaks via detailed balance and a value of $T_{\mathrm{e}}=14 \pm 3.5 \mathrm{eV}$ is found. The error in determining the free electron density and the electron temperature is $7 \%$ and $25 \%$, respectively. The small degree of ionization at a relatively high temperature is surprising and we have carried out simulations of the electron equilibration dynamics using kinetic equations [39] for two different impact ionization models: the Binary Encounter Bethe model is state of the art and in good agreement with experiments on low density plasmas [40]. The second model is based on classical electron-electron collisions [41]. We find that the classical impact ionization model shows significantly better agreement with our measurements of the free electron density and temperature. Using the non-equilibrium kinetic simulations we find further a relaxation time scale of $\sim 20 \mathrm{fs}$ of the electronic system. Therefore, the methods used to determine the hydrogen plasma parameters are justified.

A particular characteristic of the spectra is the strong suppression of the elastically scattered light, i.e. scattering stemming from electrons co-moving with the ions. This 


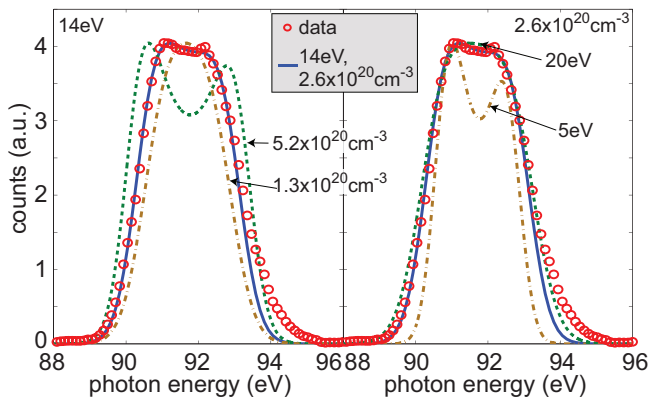

Figure 11. Measured Thomson scattering spectrum (red circles) and a best fit (blue line) of a calculated spectrum with a free electron density $n_{\mathrm{e}}=$ $(2.6 \pm 0.2) \cdot 10^{20} \mathrm{~cm}^{-3}$ and an electron temperature $T_{\mathrm{e}}=14 \pm 3.5 \mathrm{eV}$. Comparison to fits with variation in density (left graph) and in temperature (right graph) are shown.

suppression is expected for very cold ions and is due to the diffuse Debye-Waller effect. It has been predicted for WDM systems [37] and was observed in laser based experiments [38]. From the observed suppression of elastic scattering we calculate that the ion temperature must be below $0.1 \mathrm{eV}$. In conjunction with the measured electron temperature, this indicates a strong two-temperature non-equilibrium plasma during the FEL pulse duration. Details of this experiments and its analysis can be found in [15].

\section{Conclusions}

Intense, ultrashort and short wavelength FEL radiation provides a new experimental access for the investigation of solid density plasmas, in particular in the transition regime between strongly-coupled solids and ideal plasmas. First experiments using soft X-ray Thomson scattering have been successfully performed at the FLASH facility at DESY. The observed free electron densities and temperatures for the self-Thomson scattering case indicate a very fast equilibration of $\sim 20$ fs of the free electron system and thus a well-defined two-temperature non-equilibrium system of electrons and ions in the plasma [15]. Using time separated pump and probe pulses it will become possible to study time-resolved the evolution of soft X-ray or optical laser heated plasmas and to determine important EOS data for WDM plasmas. These first studies have demonstrated that the availability of diagnostics tools is crucial being adequate to characterize the incident FEL and optical laser radiation as well as their spatial and temporal overlap. Also important are reproducible and reliable sample delivery including the monitoring of the interaction process. Finally, the spectrometers to observe the scattering spectra need to have high efficiency and sufficient resolution. It is expected that in the future these techniques will also be implemented at the hard X-ray FEL facilities, providing access to even denser plasmas than accessible at FLASH.

\section{Acknowledgments}

This work was initiated by the Virtual Institute Plasma Physics using FEL radiation funded by the Helmholtz association under No. VH-VI-104 and is continued 
in the project FSP 301-FLASH funded by the German Federal Ministry for Education and Research. University Rostock acknowledges support by the Deutsche Forschungsgemeinschaft through the SFB 652 and RRF received support for the spectrometer and simulation codes from the GRK 1355 at University Hamburg. TL acknowledges DFG support under Grant No. LA 1431/2-3. The work of GG, BL and JM was supported in part by the Engineering and Physical Sciences Research Council (Grant No. EP/G007187/1) and by the Science and Technology Facilities Council of the United Kingdom. The work of TD and SHG was performed under the auspices of the U.S. Department of Energy by Lawrence Livermore National Laboratory under Contract No. DE-AC52-07NA27344. Work was also supported by Laboratory Directed Research and Development Grants No. 08-ERI-002 and No. 08-LW-004. The provision of a CCD detector by D. Riley at QUB, U.K., a He-cryostat by C.P. Schulz at MBI, Berlin, and support for access to FLASH by DESY and by the European Community under contract RII3-CT-2004-506008 (IA-SFS) are gratefully acknowledged. We finally thank all people involved at the FLASH facility for their outstanding support.

\section{References}

[1] Ackermann W et al 2007 Nature Photonics 1336

[2] Ding Y et al 2009 Phys. Rev. Lett. 102254801

[3] National Research Council 2003 Frontiers in High Energy Density Physics: The X-Games of Contemporary Science (Washington, DC: National Academies Press)

[4] Lee R W et al 2003 J. Opt. Soc. Am. B 20770

[5] Glenzer S H and Redmer R 2009 Rev. Mod. Phys. 811625

[6] Evans D E and Katzenstein J 1969 Rep. Prog. Phys. 32207

[7] García Saiz E et al 2008 Nature Physics 4940

[8] Kritcher A L et al 2008 Science 32269

[9] Glenzer S H et al 2003 Phys. Rev. Lett. 90175002

[10] Glenzer S H et al 2007 Phys. Rev. Lett. 98065002

[11] Landen O L et al 2001 Rev. Sci. Instr. 72627

[12] Saldin E L, Schneidmiller E and Yurkov M 1999 The Physics of Free Electron Lasers (Berlin: Springer-Verlag)

[13] Höll A et al 2007 High Energy Density Physics 3120

[14] Altarelli M et al 2006 European XFEL Technical Design Report (Hamburg: DESY Reports 2006-197)

[15] Fäustlin R R et al 2010 Phys. Rev. Lett. 104125002

[16] Guillot T 1999 Science $\mathbf{2 8 6} 72$

[17] Nettelmann N, Holst B, Kietzmann A, French M, Redmer R and Blaschke D 2008 Astrophys. J. 6831217

[18] Saumon D, Chabrier G and Van Horn H M, 1995 Astrophys. J. Suppl. 99713

[19] Holst B, Nettelmann N and Redmer R 2007 Contrib. Plasma Phys. 47368

[20] Holst B, Redmer R and Desjarlais M P 2008 Phys. Rev. B 77184201

[21] Fünfer E et al 1963 Phys. Rev. Lett. 5125

[22] Kunze H J et al 1964 Phys. Rev. Lett. 1142

[23] Sheffield J 1975 Plasma Scattering of Electromagnetic Radiation (New York: Academic Press)

[24] Gregori G, Glenzer S H, Rozmus W, Lee, R W and Landen O L 2003 Phys. Rev. E 67026412

[25] Tiedtke K et al 2009 New J. Phys. 11023029

[26] Meyer M et al 2006 Phys. Rev. A $74011401(\mathrm{R})$

[27] Stojanovic N et al 2010 in preparation

[28] Nordhage Ö, Li Z K, Friden C J, Norman G and Wiedner U 2005 Nucl. Instr. Meth. Phys. Res. A 546391

[29] Kühnel M, Petridis N, Winters D F A, Popp U, Dörner R, Stöhlker Th and Grisenti R E 2009 Nucl. Instr. Meth. Phys. Res. A 602311

[30] Hansson B A M and Hertz H M 2004 J. Phys. D: Appl. Phys. 373233

[31] Henke B I, Gullikson E M and Davis J C 1993 At. Data Nucl. Data Tables 54181

[32] Jasny J et al 1994 Rev. Sci. Instrum. 651631 
[33] Fäustlin R R, Zastrau U, Toleikis S, Uschmann I and Tschentscher Th 2010 JINST 5 P02004

[34] Beiersdorfer P, Crespo López-Urrutia J R, Springer P, Utter S B and Wong K L 1999 Rev. Sci. Instrum. $\mathbf{7 0} 276$

[35] Fortmann C et al 2009 High Energy Density Physics 5208

[36] Bohm D and Gross E P 1949 Phys. Rev. 751851

[37] Gregori G, Ravasio A, Höll A, Glenzer S H and Rose S J 2007 High Energy Density Physics 3 99

[38] Ravasio A et al 2007 Phys. Rev. Lett. 99135006

[39] Ziaja B, Wabnitz H, Wang F, Weckert E and Möller T 2009 Phys. Rev. Lett. 102205002

[40] Hwang W, Kim Y-K and Rudd M E 1996 J. Chem. Phys. 1042956

[41] Lieberman M A and Lichtenberg A J 1994 Principles of plasma discharges and materials processing (New York: John Wiley \& Sons)

[42] Ichimaru S, Iyetomi H and Tanaka S 1987 Phys. Rep. 14991 